\title{
Secondary cytoreductive surgery potentially improves the oncological outcomes of patients with recurrent uterine sarcomas
}

\author{
KENICHI NAKAMURA ${ }^{1 *}$, HIROAKI KAJIYAMA ${ }^{1}$, FUMI UTSUMI $^{1 *}$, SHIRO SUZUKI $^{1}$, KAORU NIIMI $^{1}$, \\ RYUICHIRO SEKIYA ${ }^{1}$, JUN SAKATA ${ }^{1}$, EIKO YAMAMOTO ${ }^{2}$, KIYOSUMI SHIBATA ${ }^{3}$ and FUMITAKA KIKKAWA ${ }^{1}$ \\ Departments of ${ }^{1}$ Obstetrics and Gynecology, and ${ }^{2}$ Healthcare Administration, Graduate School of Medicine, \\ Nagoya University, Nagoya 466-8550; ${ }^{3}$ Department of Obstetrics and Gynecology, Banbuntane \\ Hotokukai Hospital, Fujita Health University, Nagoya 454-8509, Japan
}

Received March 16, 2017; Accepted October 26, 2017

DOI: $10.3892 /$ mco.2018.1560

\begin{abstract}
Uterine sarcomas are some of the most malignant and aggressive tumor types among the gynecologic malignancies, and they are associated with a high rate of recurrence and a poor prognosis. Due to their rarity and diversity, the optimal treatment for recurrent uterine sarcomas has not yet been elucidated. The aim of the present study was to investigate the potential of secondary cytoreductive surgery (SCS) for patients with recurrent uterine sarcomas. A total of 18 patients with recurrent uterine sarcomas were retrospectively identified at the Department of Obstetrics and Gynecology, Nagoya University (Nagoya, Japan) between January 2002 and December 2015. This included 8 patients with leiomyosarcoma, 6 with carcinosarcoma, 3 with endometrial stromal sarcoma and 1 with adenosarcoma. All patients underwent primary debulking surgery as the initial treatment. In summary, 9 patients were treated with SCS when they experienced their first recurrence, and the other 9 patients were treated with non-SCS methods, including chemotherapy or radiotherapy. In the SCS group, $5 / 9$ patients had confined pelvic recurrences, 3 patients had extra-pelvic diseases, including pulmonary metastasis, and one patient had intra- and extra-pelvic recurrence. The 3-year overall survival (OS) rates were 77.8 and $11.1 \%$ in the SCS and non-SCS groups, respectively. The patients who underwent SCS experienced a significantly longer OS time compared with those in the non-SCS group $(\mathrm{P}=0.006)$. In addition, the disease-free
\end{abstract}

Correspondence to: Dr Hiroaki Kajiyama, Department of Obstetrics and Gynecology, Nagoya University Graduate School of Medicine, Tsuruma-cho 65, Showa-ku, Nagoya 466-8550, Japan

E-mail:kajiyama@med.nagoya-u.ac.jp

*Contributed equally

Abbreviations: SCS, secondary cytoreductive surgery; PFI, progression-free interval; LMS, leiomyosarcoma; ESS, endometrial stromal sarcoma; CS, carcinosarcoma

Key words: uterine sarcoma, recurrence, secondary cytoreductive surgery, disease-free interval, overall survival survival after second-line therapy was significantly longer in the SCS group than in the non-SCS group $(\mathrm{P}=0.0496)$. These findings suggest that resection of recurrent uterine sarcomas may be beneficial for the improvement of patient survival. Prospective studies with sufficient statistical power are warranted for further evaluation of the effect of SCS.

\section{Introduction}

Uterine sarcomas are relatively rare types of malignancies, comprising $1-3 \%$ of uterine malignancies (1). There are numerous histological subtypes, and it is general consensus that the most common histological subtype is leiomyosarcoma (LMS), accounting for $60-70 \%$ of all cases, followed by endometrial stromal sarcoma (ESS), $\sim 15-20 \%$ cases, worldwide (2). However, in Japan, carcinosarcoma (CS) represents $46 \%$ of uterine sarcoma diagnoses, followed by LMS (36\%) and then ESS (13\%) (3). Uterine sarcomas are some of the most malignant and aggressive tumors among the gynecologic malignancies, and often present with hematogenous metastasis in the early stages. Furthermore, they are basically refractory to treatments for relapsed tumors, such as chemotherapy and radiotherapy, resulting in a poor prognosis and a high recurrence rate. The recurrence rate has been reported to be $45-73 \%$ in LMS $(4,5)$, $37 \%$ in ESS (6) and $>50 \%$ in CS (7). The median survival times of patients with ESS, LMS and CS have been reported to be 76,31 and 28 months, respectively (3). Hysterectomy with bilateral oophorectomy is often performed as the primary standard treatment for early-stage uterine sarcomas. Conversely, the management strategy for patients with recurrent uterine sarcomas has not been well established. The efficacy of resection for lung metastasis of uterine malignancies has been demonstrated in prior studies (8-11); nevertheless, no consensus has been reached regarding the optimal treatment strategy at the time of relapse due to their rarity. The objective of the present study was to evaluate the efficacy of secondary cytoreductive surgery (SCS) for the treatment of recurrent uterine sarcomas.

\section{Patients and methods}

Patients. A retrospective study was conducted at the Department of Obstetrics and Gynecology, Nagoya University (Nagoya, 
Table I. Patients' characteristics.

\begin{tabular}{|c|c|c|c|}
\hline \multirow[b]{3}{*}{ Characteristics } & \multirow{3}{*}{$\begin{array}{c}\text { Total } \\
\mathrm{n}\end{array}$} & \multicolumn{2}{|c|}{ SCS } \\
\hline & & Yes & No \\
\hline & & $\mathrm{n}(\%)$ & $\mathrm{n}(\%)$ \\
\hline Total & 18 & 9 & 9 \\
\hline Median age at recurrence & 61.5 & 62.0 & 54 \\
\hline Range (years) & $33-71$ & $33-71$ & $37-68$ \\
\hline Median follow-up (Mo) & 29.7 & 90.5 & 22.9 \\
\hline Range (months) & $(2.7-358.3)$ & $(11.1-358.3)$ & $(2.7-58.9)$ \\
\hline \multicolumn{4}{|l|}{ Histological type } \\
\hline LMS & 8 & $3(33.3)$ & $5(55.6)$ \\
\hline $\mathrm{CS}$ & 6 & $2(22.2)$ & $4(44.4)$ \\
\hline ESS & 3 & $3(33.3)$ & $0(0)$ \\
\hline Others & 1 & $1(11.1)$ & $0(0)$ \\
\hline \multicolumn{4}{|l|}{ FIGO stage } \\
\hline I & 9 & $7(77.8)$ & $2(22.2)$ \\
\hline II & 0 & $0(0)$ & $0(0)$ \\
\hline III & 6 & $2(22.2)$ & $4(44.4)$ \\
\hline IV & 3 & $0(0)$ & $3(33.3)$ \\
\hline \multicolumn{4}{|l|}{ Initial surgery } \\
\hline $\mathrm{ATH}+\mathrm{BSO}$ & 13 & $7(77.8)$ & $6(66.7)$ \\
\hline $\mathrm{ATH}+\mathrm{BSO}+\mathrm{PLN}$ & 4 & $2(22.2)$ & $2(22.2)$ \\
\hline Tumor resection & 1 & $0(0)$ & $1(11.1)$ \\
\hline \multicolumn{4}{|c|}{ Residual tumor at primary surgery } \\
\hline Complete & 16 & $8(88.9)$ & $8(88.9)$ \\
\hline Incomplete & 2 & $1(11.1)$ & $1(11.1)$ \\
\hline \multicolumn{4}{|c|}{ No. of total chemotherapy regimens } \\
\hline 0 & 2 & $2(22.2)$ & $0(0)$ \\
\hline 1 & 5 & $1(11.1)$ & $4(44.4)$ \\
\hline 2 & 11 & $6(66.7)$ & $5(55.6)$ \\
\hline \multicolumn{4}{|c|}{ Progression/recurrence-free interval } \\
\hline$\geq 6$ months & 12 & $8(88.9)$ & $4(44.4)$ \\
\hline$<6$ months & 6 & $1(11.1)$ & $5(55.6)$ \\
\hline \multicolumn{4}{|l|}{ Outcomes } \\
\hline NED & 3 & $3(33.3)$ & 0 \\
\hline AWD & 2 & $2(22.2)$ & 0 \\
\hline DOD & 13 & $4(44.4)$ & $9(100)$ \\
\hline
\end{tabular}

SCS, secondary cytoreductive surgery; LMS, leiomyosarcoma; ESS, endometrial stromal sarcoma; CS, carcinosarcoma; FIGO, International Federation of Gynecology and Obstetrics; ATH, abdominal total hysterectomy; BSO, bilateral salpingo-oophorectomy; PLN, pelvic lymphoadenectomy; NED, no evidence of disease; AWD, alive with disease; DOD, dead of disease.

Japan) using a computerized database, and 35 females with pathologically verified sarcoma who underwent primary or recurrent treatment between January 2002 and December 2015 were identified. In the 35 patients with uterine sarcomas, 18 patients who experienced recurrent disease in the study period were included in the present study. Clinicopathological data from patients' medical records were collected, including the age, pathological diagnosis, stage, initial treatment, details of treatment, relapse information, follow-up and survival data.
The tumor stage was based on the criteria of the International Federation of Gynecology and Obstetrics (2008). Written informed consent to collect and use their data for clinical research was acquired from all patients. The study protocol was approved by the Ethics Committee of Nagoya University Graduate School of Medicine (approval no. 2013-0078).

Follow-up. At the conclusion of the initial treatment, all patients underwent a strict follow-up regime, consisting of the 
following clinical checkups: Pelvic examination, ultrasonographic scans, magnetic resonance imaging (MRI), periodic computed tomography (CT) and/or positron emission tomography (PET). Recurrence was defined as the presence of a relapsed tumor based on the results of $\mathrm{CT}$ and/or MRI and/or PET-CT and/or ultrasound.

Statistical analysis. Patient data were censored at the time of mortality or final contact due to causes other than uterine sarcoma or disease-associated complications. The distributions of clinicopathological events were evaluated using Fisher's exact test. Kaplan-Meier curves were generated from the survival data. All statistical analyses were performed with EZR version 1.32 (Saitama Medical Center, Jichi Medical University, Saitama, Japan), which is a graphical user interface for R (The R Foundation for Statistical Computing, Vienna, Austria). Specifically, EZR is a modified version of the $\mathrm{R}$ commander designed to add statistical functions frequently used in biostatistics. A value of $\mathrm{P}<0.05$ was considered to indicate a statistically significant difference.

\section{Results}

Patients' characteristics. Details of the patients' characteristics are listed in Table I. During the study period, a total of 18 patients with recurrent uterine sarcomas were identified. Of these, $8(44.4 \%), 6(33.3 \%)$ and $3(16.7 \%)$ patients had a diagnosis of LMS, CS and ESS, respectively. The one remaining (5.6\%) patient had another type of uterine sarcoma. The median age at the time of SCS was 61.5 years (range, 33-71 years) and the median follow-up time, including deceased patients, was 29.7 months [range, 2.7-358.3 months (due to initial treatment in 1986 for one case)]. A total of 9 (50.0\%), 6 (33.3\%) and 3 (16.7\%) patients were diagnosed with FIGO stage I, III and IV, respectively. All patients underwent primary debulking surgery as the initial treatment. A total of 17 patients (88.9\%) underwent at least hysterectomy-including surgery, and 4 patients received pelvic lymphadenectomy as well as hysterectomy (22.2\%). The pathological diagnosis of the 4 patients who underwent pelvic lymphadenectomy was CS in all cases. The tumor was completely removed by primary surgery in 16 patients (88.9\%). Among the 18 patients with recurrent uterine sarcomas, SCS was performed in 9 patients. The SCS group contained 3 patients with ESS, while there were no patients with this histology in the non-SCS group. Additionally, the rate of having a progression-free interval (PFI) $>6$ months was higher in the SCS patient group than in the non-SCS patient group [SCS group, 88.9\% (8/9) vs. non-SCS group, $44.4 \%$ (4/9); $\mathrm{P}=0.0231$ (Fisher's exact test)].

Analysis of treatment outcomes and recurrence. Table II lists the recurrence sites for 18 patients who experienced recurrent uterine sarcomas. In the SCS group, 5/9 patients had confined pelvic recurrences, 3 patients had extra-pelvic diseases, including pulmonary metastasis, and one patient had intra- and extra-pelvic recurrence. Conversely, of nine patients in the non-SCS group, 4 had confined pelvic recurrences, 2 had extra-pelvic diseases, including pulmonary metastasis, and 3 had intra- and extra-pelvic recurrence. The details of the SCS are presented in Table III. It is notable that 4 patients
Table II. The site of recurrence in patients with or without SCS.

\begin{tabular}{|c|c|c|c|}
\hline \multirow[b]{3}{*}{ Recurrence site } & \multirow{3}{*}{$\begin{array}{c}\text { Total } \\
\mathrm{n}\end{array}$} & \multicolumn{2}{|c|}{ SCS } \\
\hline & & Yes & No \\
\hline & & $\mathrm{n}(\%)$ & $\mathrm{n}(\%)$ \\
\hline Intrapelvic recurrence & 9 & $5(55.6)$ & $4(44.4)$ \\
\hline Extrapelvic recurrence & 6 & $3(33.3)$ & $3(33.3)$ \\
\hline Both & 3 & $1(11.1)$ & $2(22.2)$ \\
\hline
\end{tabular}

SCS, secondary cytoreductive surgery.

had a number of surgeries following SCS, the highest number being 8 further surgical procedures. Furthermore, no serious complications of the SCS were observed in the current study. As salvage therapy for 9 patients treated without SCS, 8 underwent subsequent chemotherapy and 1 patient was treated with radiotherapy.

The re-recurrence rate and PFI following SCS are presented in Table IV. Although the patient number was limited, relatively good disease control was observed in patients with LMS and CS. The 3-year OS rates were 77.8 and $11.1 \%$ in the SCS and non-SCS groups, respectively. As presented in Fig. 1, patients who underwent SCS experienced a significantly longer OS than those who did not receive SCS $(\mathrm{P}=0.006)$. Additionally, the PFI post-SCS was significantly longer in the SCS group compared with that in the non-SCS group ( $\mathrm{P}=0.0496$; Fig. 2$)$.

\section{Discussion}

Due to the rarity and heterogeneity of uterine sarcomas, optimal management strategies have not yet been established (12). Furthermore, despite the application of appropriate primary therapy, uterine sarcomas often behave in an aggressive and malignant fashion with a high risk of recurrence and poor prognosis. The optimal treatment for recurrent uterine sarcomas remains unclear. These tumors are relatively chemoand/or radio-resistant, and certain studies have previously evaluated the effectiveness of SCS in patients with recurrent uterine sarcomas $(11,13,14)$.

In the present study, it was demonstrated that patients with recurrent uterine sarcomas who underwent the SCS had longer overall survival times and disease-free intervals following the initial relapse, when compared with those treated with chemotherapy or radiotherapy alone. SCS for patients who have pulmonary recurrent disease has been reported to lead to improved survival, particularly in those with unilateral disease, a smaller number of resected metastases $(<3)$, a smaller tumor $(<3 \mathrm{~cm})$ and a longer DFI $(<6$, or 12 months) $(8-10)$. However, studies on SCS for extra-pulmonary recurrences of uterine sarcomas are limited, and have only small sample sizes $(4,11,14-17)$. Leitao et al (14) reported that optimal surgical resection may improve the survival of patients with either pulmonary or extra-pulmonary metastases, after retrospectively surveying 41 patients with recurrent LMS (including 28 patients who had non-thoracic recurrences). Yoshinaga et al (17) concluded that patient survival was 
Table III. Details of secondary cytoreductive surgery.

\begin{tabular}{|c|c|c|c|c|c|c|c|c|c|c|}
\hline \multirow[b]{2}{*}{ Case } & \multirow[b]{2}{*}{ Histology } & \multirow[b]{2}{*}{ Rec. site } & \multirow[b]{2}{*}{$\begin{array}{c}\text { No. } \\
\text { metastasis }\end{array}$} & \multicolumn{3}{|c|}{ SCS } & \multirow[b]{2}{*}{ RT } & \multirow[b]{2}{*}{$\begin{array}{l}\text { No. of } \\
\text { surgery }\end{array}$} & \multirow[b]{2}{*}{ Outcomes } & \multirow[b]{2}{*}{$\begin{array}{c}\text { PFI } \\
\text { (months) }\end{array}$} \\
\hline & & & & Surgery & $\begin{array}{l}\text { Time } \\
(\min )\end{array}$ & $\begin{array}{c}\text { Blood } \\
\text { loss }(\mathrm{ml})\end{array}$ & & & & \\
\hline 1 & Others & Pelvis & 14 & Met & 315 & 2,007 & Incomplete $^{\mathrm{a}}$ & 1 & DOD & 3.1 \\
\hline 2 & $\mathrm{CS}$ & Pelvis & 8 & PM, Col & 389 & 1,940 & Complete $^{\mathrm{b}}$ & 2 & DOD & 6.9 \\
\hline 3 & LS & Omentum & 1 & BSO, OM & $\mathrm{N} / \mathrm{A}$ & $\mathrm{N} / \mathrm{A}$ & Complete & 9 & DOD & 8.6 \\
\hline 4 & LS & Pelvis & 1 & Met & 103 & 147 & Complete & 1 & NED & 63.7 \\
\hline 5 & ESS & Lung & 6 & Met & 127 & 20 & Complete & 3 & NED & 48.0 \\
\hline 6 & ESS & Lung, pelvis & 3 & Met & 1,073 & 6,362 & Complete & 3 & AWD & 31.7 \\
\hline 7 & ESS & Inguinal LN & 1 & Met & 41 & 28 & Residual $^{\mathrm{c}}$ & 1 & DOD & 14.4 \\
\hline 8 & $\mathrm{CS}$ & Pelvis & 1 & Met, Col & 239 & 668 & Complete & 1 & DOD & 18.2 \\
\hline 9 & LMS & Pelvis & 2 & Met, Col & 420 & 650 & Complete & 1 & NED & 43.2 \\
\hline
\end{tabular}

${ }^{\mathrm{a} N o}$ macroscopic RT; ${ }^{\mathrm{b} T}<2 \mathrm{~cm}$ in diameter; ${ }^{\mathrm{R} T} \geq 2 \mathrm{~cm}$ in diameter. SCS, secondary cytoreductive surgery; PFI, progression-free interval; RT, residual tumor; LMS, leiomyosarcoma; ESS, endometrial stromal sarcoma; CS, carcinosarcoma; Met, metastasectomy; Col, colectomy; NED, no evidence of disease; AWD, alive with disease; DOD, died of disease.

Table IV. Frequency of recurrence after secondary cytoreduction surgery.

\begin{tabular}{lcc}
\hline Histology & $\begin{array}{c}\text { Frequency of } \\
\text { recurrence, n }(\%)\end{array}$ & $\begin{array}{c}\text { Median } \\
\text { PFI (months) }\end{array}$ \\
\hline LMS & $1 / 3(33.3)$ & 8.6 \\
CS & $2 / 2(100)$ & $12.5(9.0-16.0)$ \\
ESS & $2 / 3(66.7)$ & $3.8(2.3-5.4)$ \\
Other & $1 / 1(100)$ & 3.1 \\
Total & $6 / 9(66.7)$ & $9.7(2.3-16.0)$ \\
\hline
\end{tabular}

PFI, progression-free interval after secondary surgery; LMS, leiomyosarcoma; ESS, endometrial stromal sarcoma; CS, carcinosarcoma.

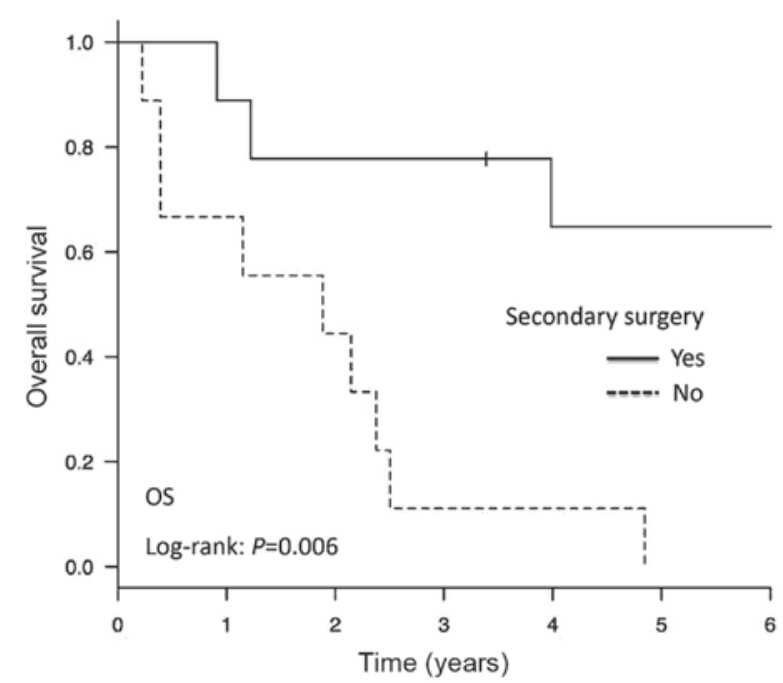

Figure 1. Kaplan-Meier curves show the overall survival following salvage treatments for recurrent uterine sarcomas. The solid line represents patients who underwent SCS $(n=9)$. The dashed line represents patients who underwent non-SCS treatment, including salvage chemotherapy and/or radiotherapy ( $\mathrm{n}=9 ; \mathrm{P}=0.006)$. $\mathrm{SCS}$, secondary cytoreductive surgery.

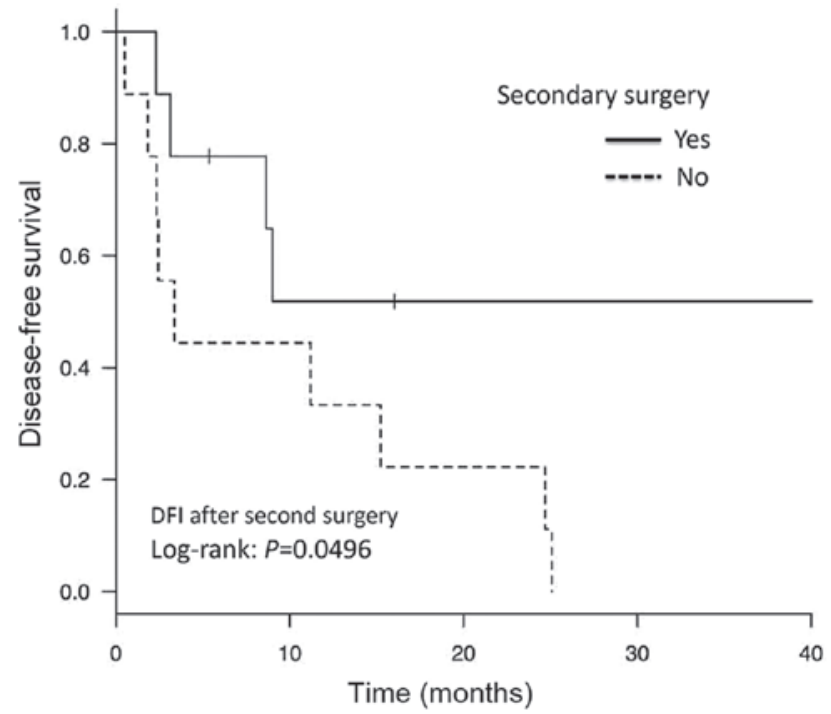

Figure 2. Kaplan-Meier curves estimates of the disease/progression-free interval after salvage treatment for recurrent uterine sarcomas. The solid line represents patients who underwent the SCS $(n=9)$. The dashed line represents patients who underwent non-SCS treatment, including salvage chemotherapy and/or radiotherapy $(\mathrm{n}=9 ; \mathrm{P}=0.0496)$. SCS, secondary cytoreductive surgery.

improved by the resection of pulmonary or intrapelvic metastases of uterine sarcomas. Nevertheless, this report included only 8 patients, 3 of whom had extra-pulmonary recurrences. According to a review by Giuntoli et al (4) that analyzed 208 patients with recurrent LMS, SCS is associated with the improvement of disease-specific survival from the first relapse, unlike chemotherapy or radiotherapy.

In the current study, 8/9 patients in the SCS group had extra-pulmonary metastases. Although 4 of these had multiple metastases (the number of metastases, 2-14), SCS may contribute to prolonged survival. Hoang et al (11) reported that single tumor recurrence was significantly correlated with a longer survival time, when compared with that of patients with $>2$ tumors. 
Based on the current examination, the resection of recurrent tumors may improve survival, even in patients with multiple extra-pulmonary diseases derived from uterine sarcomas. As aforementioned, the optimal indication regarding the number of metastases in candidate has yet to be clarified. However, in this study, no severe complications of multiple metastasectomy were observed. Therefore, SCS may be considered even for patients with multiple recurrences, if there are no other favorable alternatives and optimal resection is considered possible.

In the current study, the re-recurrence rate was 67\% (6/9) in patients treated with SCS, while all patients $(9 / 9 ; 100 \%)$ treated without SCS experienced relapse. It may be considered that SCS is a superior management procedure to chemo- or radiotherapy for recurrent uterine sarcoma. Nevertheless, the limitations of the current study are those associated with any retrospective study: insufficient patient numbers, the possibility of selection bias and treatment heterogeneity. Thus, it is possible the results reflect the fact that patients with a favorable condition for SCS demonstrated a better oncologic outcome. The largest limitation of the present study was that only 18 patients with recurrent sarcoma were available. This small-scale patient cohort was based on the actual clinical situation that recurrent uterine sarcomas are rare. A large-scale additional study verifying the current findings is desirable, involving more patients from multiple institutions. Further limitations of the current report are those associated with any retrospective study. Indeed, the treatment protocol for uterine sarcomas, including the regimen of salvage chemotherapy, the number of courses, doses, criteria of surgery, diagnostic and surgical procedures were heterogeneous. In particular, the development of surgical devices and perioperative management is frequent and evident currently. Furthermore, the advancement of imaging modalities, including MRI, new-generation ultrasound and PET-CT, has contributed to surgical decisions. The possibilities that these factors may influence the treatment and disease outcomes of patients with recurrent uterine sarcomas must be considered. It is possible the current findings provide essential information on how to develop better treatments for this type of tumor.

In conclusion, the results of the current investigation support the consideration of SCS for patients with recurrent uterine sarcomas. Several reports have recently demonstrated that new-generation anticancer agents, including bevacizumab, pazopanib, eribulin and trabectedin, are effective for recurrent LMS (18-21). Comparison of SCS with these treatments could be useful. Irrespective of this, the rates of mortality and relapse remain high and further studies are required.

\section{Competing interests}

The authors declare that they have no competing interests.

\section{References}

1. Park JY, Kim DY, Suh DS, Kim JH, Kim YM, Kim YT and Nam JH: Prognostic factors and treatment outcomes of patients with uterine sarcoma: Analysis of 127 patients at a single institution, 1989-2007. J Cancer Res Clin Oncol 134: 1277-1287, 2008.

2. Burghaus S, Halmen S, Gass P, Mehlhorn G, Schrauder MG, Lux MP, Renner SP, Beckmann MW, Hein A and Thiel FC: Outcome and prognosis in uterine sarcoma and malignant mixed Mullerian tumor. Arch Gynecol Obstet 294: 343-351, 2016.
3. Fujita H, Adachi S, Kigawa J, Sugiyama T and Takeuchi S: A clinicopathlogical study of uterine sarcoma in last decade-a retrospective study of KCOG/USSG inter group study. Adv Obstet Gynecol 56: 463-465, 2004.

4. Giuntoli RL II, Garrett-Mayer E, Bristow RE and Gostout BS: Secondary cytoreduction in the management of recurrent uterine leiomyosarcoma. Gynecol Oncol 106: 82-88, 2007.

5. Mayerhofer K, Obermair A, Windbichler G, Petru E, Kaider A, Hefler L, Czerwenka K, Leodolter S and Kainz C: Leiomyosarcoma of the uterus: A clinicopathologic multicenter study of 71 cases. Gynecol Oncol 74: 196-201, 1999.

6. Leath CA III, Huh WK, Hyde J Jr, Cohn DE, Resnick KE, Taylor NP, Powell MA, Mutch DG, Bradley WH and Geller MA: A multi-institutional review of outcomes of endometrial stromal sarcoma. Gynecol Oncol 105: 630-634, 2007.

7. Callister M, Ramondetta LM, Jhingran A, Burke TW and Eifel PJ: Malignant mixed mullerian tumors of the uterus: Analysis of patterns of failure, prognostic factors and treatment outcome. Int J Radiat Oncol Biol Phys 58: 786-796, 2004.

8. Anraku M, Yokoi K, Nakagawa K, Fujisawa T, Nakajima J, Akiyama H, Nishimura Y and Kobayashi K; Metastatic Lung Tumor Study Group of Japan: Pulmonary metastases from uterine malignancies: Results of surgical resection in 133 patients. J Thorac Cardiovasc Surg 127: 1107-1112, 2004.

9. Anderson TM, McMahon JJ, Nwogu CE, Pombo MW Urschel JD, Driscoll DL and Lele SB: Pulmonary resection in metastatic uterine and cervical malignancies. Gynecol Oncol 83: 472-476, 2001.

10. Levenback C, Rubin SC, McCormack PM, Hoskins WJ, Atkinson EN and Lewis JL Jr: Resection of pulmonary metastases from uterine sarcomas. Gynecol Oncol 45: 202-205, 1992.

11. Hoang HL, Ensor K, Rosen G, Leon Pachter H and Raccuia JS: Prognostic factors and survival in patients treated surgically for recurrent metastatic uterine leiomyosarcoma. Int J Surg Oncol 2014: 919323, 2014.

12. Reed NS: The management of uterine sarcomas. Clin Oncol (R Coll Radiol) 20: 470-478, 2008.

13. Burt BM, Ocejo S, Mery CM, Dasilva M, Bueno R, Sugarbaker DJ and Jaklitsch MT: Repeated and aggressive pulmonary resections for leiomyosarcoma metastases extends survival. Ann Thorac Surg 92: 1202-1747, 2011.

14. Leitao MM, Brennan MF, Hensley M, Sonoda Y, Hummer A, Bhaskaran D, Venkatraman E, Alektiar K and Barakat RR: Surgical resection of pulmonary and extrapulmonary recurrences of uterine leiomyosarcoma. Gynecol Oncol 87: 287-294, 2002.

15. Chen H, Pruitt A, Nicol TL, Gorgulu S and Choti MA: Complete hepatic resection of metastases from leiomyosarcoma prolongs survival. J Gastrointest Surg 2: 151-155, 1998.

16. Korets SB and Curtin JP: Surgical options for recurrent uterine sarcomas. Am Soc Clin Oncol Educ Book: 362-366, 2012.

17. Yoshinaga M, Togami S, Tsuji T, Fukamachi N, Kamio M, Yanagi M and Douchi T: Clinical outcome of metastatic uterine leiomyosarcoma and carcinosarcoma in a single institute. J Obstet Gynaecol Res 33: 818-822, 2007.

18. Benson C, Ray-Coquard I, Sleijfer S, Litière S, Blay JY, Le Cesne A, Papai Z, Judson I, Schöffski P, Chawla S, et al: Outcome of uterine sarcoma patients treated with pazopanib: A retrospective analysis based on two European organisation for research and treatment of cancer (EORTC) soft tissue and bone sarcoma group (STBSG) clinical trials 62043 and 62072. Gynecol Oncol 142: 89-94, 2016.

19. Han Y, Li S, Holt HK and Wu L: Curative effect of bevacizumab combined with chemotherapy in advanced or recurrent uterine sarcoma. Mol Clin Oncol 4: 245-248, 2016.

20. Martin-Broto J, Pousa AL, de Las Peñas R, García Del Muro X, Gutierrez A, Martinez-Trufero J, Cruz J, Alvarez R, Cubedo R, Redondo A, et al: Randomized Phase II study of trabectedin and doxorubicin compared with doxorubicin alone as first-line treatment in patients with advanced soft tissue sarcomas: A spanish group for research on sarcoma study. J Clin Oncol 34: 2294-2302, 2016.

21. Schöffski P, Chawla S, Maki RG, Italiano A, Gelderblom H, Choy E, Grignani G, Camargo V, Bauer S, Rha SY, et al: Eribulin versus dacarbazine in previously treated patients with advanced liposarcoma or leiomyosarcoma: A randomised, open-label, multicentre, phase 3 trial. Lancet 387: 1629-1637, 2016. 